\title{
Metadatos y audiovisual: iniciativas, esquemas y estándares
}

\author{
Juan Antonio POLO CARRIÓN \\ Canal Extremadura Radio \\ japolcar@alcazaba.unex.es \\ Jorge CALDERA SERRANO \\ Universidad de Extremadura \\ jcalser@alcazaba.unex.es \\ Inés Carmen POVEDA LÓPEZ \\ Canal Extremadura Televisión \\ icpovlop@alcazaba.unex.es
}

Recibido: 04/04/2011

Aceptado: 10/05/2011

\section{RESUMEN}

Se presentan las más destacadas iniciativas, esquemas y estándares de metadatos relacionados con el ámbito de la gestión documental, haciendo especial hincapié en aquellos que pudiera ser útiles para ser adaptados en los archivos audiovisuales televisivos. Se enumeran y describen las iniciativas modelo IFLA-FRBR, INDECS, SMEF, Ontología ABC, Video Active, ECHO, Open Video Digital Library. Igualmente se analizan los estándares AAF, Dublin Core, Lista de datos mínimos de la FIAT-IFA, METS, MPEG, MXF, P-FRA, P-Meta, SMIL, SMPTE, TV-ANYTIME y UMID.

Palabras clave: Metadato, Audiovisual, Estándares de metadatos, Gestión documental, televisión

\section{The metadata and audiovisual: initiatives, schemes and standards}

\begin{abstract}
It presents the most important initiatives, schemes and metadata standards related to the area of document management, with particular emphasis on those that might be useful to be adapted into television audiovisual archives. It lists and describes the IFLA-FRBR model initiatives, INDECS, SMEF, Ontology ABC, Video Active, ECHO, Open Video Digital Library. Also discussed AAF standards, Dublin Core, Minimum Data List FIAT-IFA, METS, MPEG, MXF, P-FRA, P-Meta, SMIL, SMPTE, TV-Anytime and UMID.
\end{abstract}

Key words: Metadata Audiovisual, Metadata Standards, Document Management, television 


\section{ESTADO DE LA CUESTIÓN}

$\mathrm{Al}$ igual que ha pasado con la documentación tradicional, la audiovisual ha sufrido cambios motivado por el avance de las nuevas tecnologías. Quizás estos cambios son más notables en la documentación audiovisual, ya que el uso de Internet unido a los procesos de digitalización ha hecho que las empresas del sector asuman los retos que plantean los nuevos modos de producción y difusión de la información audiovisual que permiten su adaptación en el nuevo entorno tecnológico.

Estos retos surgen como consecuencia de las características tanto del proceso de creación y gestión como del propio documento audiovisual, y que podemos resumir en migración de colecciones, normas de interoperabilidad para las redes, compatibilidad de los sistemas media, nuevos servicios a los clientes, etc. (De Jong, 2003). Además aquellas unidades con problemas de conservación han visto en la digitalización su salvación (López, Sánchez, Pérez, 2003). Es decir, aquellas unidades que cuentan con un gran fondo, por medio de los procesos de digitalización del mismo, pueden almacenar documentación que por falta de espacio no podrían conservar. Esto supone un avance tanto en la forma de almacenar como la de trabajar, ya que al estar en soporte digital, es más fácil transferir o recuperar la información que se necesite.

En esta nueva forma de trabajo los sistemas automatizados van a tener un papel importante. Todos los elementos del proceso (sonidos, imágenes fijas o en movimiento, traducciones, otro tipo de información, etc) serán parte de una base de datos que servirá de sustento a la información y la documentación, la producción y la difusión, el archivo y el acceso a los materiales.

Así, dentro de esta manera de trabajar, convergerán los distintos grupos profesionales que coexisten en un sistema de información documental, como por ejemplo los especialistas en información, realizadores de programas, montados, productores, etc, y serán ellos quienes creen las características del sistema, por lo que este deberá ser entendido por todos e interpretable de manera única.

Como decimos, se ha producido un aumento de la información audiovisual, lo que se traduce en un aumento de la producción, distribución y archivo de programas, lo que nos lleva a soluciones como la llamada producción sin cinta. Que no es más que la producción, distribución y archivo de la información audiovisual de forma digital. Por ejemplo, en la actualidad existen modelos de cadenas de televisión u acontecimientos (tales como conciertos, retransmisiones deportivas, actos políticos, etc.) que se emiten digitalmente. Esta información circulará de manera permanente por la red, por lo que se deberá de tratar de forma que podamos identificarla en cualquier momento ejerciendo un control sobre la misma. Este consiste en una estructura de información precisa que dirige el almacenamiento, distribución, producción y archivo de datos digitales y, al mismo tiempo, define la forma y el contenido de estos elementos mediante protocolos, normas y estándares (De Jong, 2003).

Si nos centramos en las actividades relacionadas con el análisis de contenido podemos hablar de los metadatos. Orozco García-Mayorca (2001?) comenta que el análisis documental de imágenes en movimiento (que conlleva el diseño de los metadatos) "consiste en contemplar tanto la descripción sintética de la obra 
(catalogación) como la descripción de las secuencias o de los planos (análisis de contenido), para mantener la integridad del documento".

En el ámbito audiovisual el concepto de metadato tiene diversas interpretaciones según el colectivo que los defina (De Jong, 2003):

\begin{tabular}{|l|l|}
\hline $\begin{array}{l}\text { Técnicos de } \\
\text { información }\end{array}$ & $\begin{array}{l}\text { Aquello que incumbe a los sistemas de información, incluyendo } \\
\text { los modelos de datos y la arquitectura técnica. }\end{array}$ \\
\hline $\begin{array}{l}\text { Una empresa de } \\
\text { difusión }\end{array}$ & $\begin{array}{l}\text { Información que se centra en la identificación y análisis del } \\
\text { contenido media. }\end{array}$ \\
\hline $\begin{array}{l}\text { Producción } \\
\text { audiovisual }\end{array}$ & $\begin{array}{l}\text { Datos lógicos y su modo de clasificación, en tanto que están } \\
\text { relacionados con los materiales de programas y la manera en } \\
\text { que se procesan. }\end{array}$ \\
\hline $\begin{array}{l}\text { Analistas de la } \\
\text { información }\end{array}$ & $\begin{array}{l}\text { La información en tanto que es la estructura del propio } \\
\text { metadato la que nos remite al contenido digital. }\end{array}$ \\
\hline
\end{tabular}

De Jong comenta que no hay consenso a la hora de establecer una única definición debido a la "oposición entre los especialistas técnicos y los especialistas de la información". Mientras los primeros insisten en el contenido de las listas de metadatos, los segundos buscan cómo se estructuran.

El uso de los metadatos en el entorno audiovisual se presenta diferente a como los usamos en la documentación escrita.

"Esto es debido a la complejidad de los procesos de producción, edición, difusión y archivo dentro del campo de la documentación audiovisual es muy superior y cuenta con muchas más variables que en el caso de la escrita" (López, Sánchez, Pérez, 2003).

Además hay una mayor diversidad de formatos y medios que dificulta la recuperación de la información, ya que búsquedas con correspondencia exacta no ayudan al usuario cuando tiene una base de datos con diferentes materiales. Por otra parte, debido al tamaño de las colecciones audiovisuales, búsquedas basadas en el contenido no funcionan bien, ya que se necesitan otras informaciones para mejorar su recuperación.

En el tratamiento de la información audiovisual se generan diferentes datos añadidos que acompañan al contenido del documento y cuyo interés es a veces igual que el propio documento ya que nos permiten facilitar procesos relacionados con la transferencia, la distribución interna y externa y el almacenamiento. Es decir, los documentos audiovisuales contienen un tipo de información implícita que constituye un tipo de metadatos. Esta información es parte del vídeo o del audio y puede necesitarse para ser recuperada.

Otro aspecto importante de estos metadatos es que sirven de nexo de unión entre los sistemas actuales o antiguos y los nuevos que surjan, ya que en ambos el fin de estos metadatos será el mismo, que no es otro que el de describir la información de un documento, además de favorecer el intercambio de información digital entre distintas plataformas. 
Centrándonos en el ciclo de vida de los metadatos, podemos afirmar lo siguiente: Referente al momento de creación, pueden asignarse de tres modos:

- Mediante la indización automática de elementos del contenido (extracción de palabras clave de la locución).

- Mediante la incorporación semiautomática de información externa (el texto de un catálogo).

- Mediante la información incluida en los procesos de creación y codificación de las tomas (fecha y hora registrada por la cámara).

Sobre su uso, nos facilitan la navegación, visualización y la recuperación de los documentos audiovisuales indizados con ellos. Otro aspecto a tener en cuenta es que si contamos con una base de datos con metadatos de diversos tipos, cada departamento de la unidad puede otorgar sus propios metadatos según sus intereses o adaptar los ya creados y hacer más completa la base de datos según los fines de cada departamento: así podemos encontrarnos con metadatos basados en el contenido (con información sobre la duración, el lugar de grabación, descriptores otorgados, etc.) o metadatos orientados a describir los materiales (formato utilizado, estado de conservación, etc.).

Una vez visto qué es lo que podemos hacer con los metadatos, tendremos que ver cómo se van a conservar. Su almacenamiento puede ser de diversos tipos:

- Separados del contenido del documento al que describen.

- Almacenados junto al documento.

En ambos casos los metadatos deben de llevar un mantenimiento para mejorar la calidad de la base de datos y facilitar así la recuperación de la información. Este mantenimiento puede llevarse a cabo por cada uno de los departamentos, que serán responsables de sus propios metadatos, o de manera centralizada. De todas formas deben de establecerse normativas relacionadas con la creación y control de los metadatos.

Siguiendo comentando aspectos relacionados con los metadatos audiovisuales podemos ver que hay distintas clasificaciones realizadas, entre ellas destaca la realizada por De Jong (2003) quien agrupa a los metadatos según el tipo de medio, el procesado de un determinado medio y el contenido. 


\begin{tabular}{|l|l|}
\hline $\begin{array}{l}\text { Según el } \\
\text { tipo de } \\
\text { medio }\end{array}$ & $\begin{array}{l}\text { Puede ser el medio con le que se relacionan o del que derivan; ejemplos } \\
\text { serían la frecuencia de muestreo, textura o tipo de caracteres. A mayor } \\
\text { especificidad de los metadatos, más propios serán. }\end{array}$ \\
\hline $\begin{array}{l}\text { Según el } \\
\text { procesado. }\end{array}$ & $\begin{array}{l}\text { No tienen que ver con el contenido, sino con las características } \\
\text { necesarias para poder procesar el documento. Estos metadatos son parte } \\
\text { de los mecanismos que vinculan los ficheros audiovisuales con los } \\
\text { correspondientes metadatos para dirigir e intercambiar este contenido } \\
\text { entre los miembros del sistema. }\end{array}$ \\
\hline $\begin{array}{l}\text { Según el } \\
\text { contenido }\end{array}$ & $\begin{array}{l}\text { Son los derivados del contenido del documento sin importar el tipo de } \\
\text { medio o proceso. Se dividen en metadatos basados directamente en el } \\
\text { contenido (como los índices) o metadatos descriptivos del contenido, } \\
\text { que a su vez se subdividen en: } \\
\text { Metadatos descriptivos objetivos: autor, título, duración } \\
\text { del programa, fechas de producción y catalogación. } \\
\text { Metadatos temáticos: descripción del contenido o parte } \\
\text { de él, del tema y del significado. } \\
\text { Metadatos adicionales: otra información sobre el conte- } \\
\text { nido de manera subjetiva. }\end{array}$ \\
\hline
\end{tabular}

Kashyap (1997) distingue, al igual que De Jong, entre metadatos independientes del contenido y dependientes. Pero además añade otra tipología más, como son los metadatos independientes y específicos del dominio.

Los primeros los podemos definir como aquellos que no dependen del área de aplicación o temática del documento, por ejemplo definiciones de documentos SGML. Por su parte, los metadatos específicos del dominio son los relacionados con la temática y suelen contar con tesauros específicos, ontologías o diccionarios de autoridad.

\section{INICIATIVAS DE METADATOS Y AUDIOVISUAL}

A continuación pasamos a describir algunas de las iniciativas más importantes. De todas las iniciativas emprendidas hemos elegido la desarrollada por la IFLA llamada FRBR y centrada en el ámbito de las bibliotecas y archivos, aunque en nuestro trabajo se expondrá una adaptación de dicho modelo al ámbito de los archivos audiovisuales; SMEF centrado en el entorno de la producción; <indecs> para la gestión de los derechos y el modelo ABC australiano. Por último comentaremos brevemente el proyecto norteamericano destinado a dar acceso libre a vídeos creados por las agencias gubernamentales y que utiliza metadatos para la descripción y acceso a esos materiales. 


\section{El modelo IFLA-FRBR (Requerimientos Funcionales para Registros Bibliográficos)}

Fue definido por la IFLA en 1998 e incluye al registro bibliográfico como una agregación de datos que se asocian con las entidades de los catálogos. Este modelo tiene una estructura multinivel y jerárquica que permite remodelarse para adaptarse a unas necesidades concretas. La metodología llevada a cabo comienza por aislar las entidades que son objeto de interés para los usuarios de los registros bibliográficos, para luego identificar las características asociados con cada entidad y las relaciones entre ellas. Estas entidades se dividen en tres grupos: las obras, sus responsables e información adicional que normalmente se suele usar como las materias de las obras.

FRBR sirvió de base al modelo ECHO (European Chronicles On Line) cuyo fin era definir un nuevo modelo abierto e interoperable capaz de representar los múltiples aspectos de los recursos audiovisuales (Ríos Hilario, 2006).

\section{$<I N D E C S>$}

Basado en el modelo anterior surge para describir e identificar la propiedad intelectual y las personas relacionadas con la misma por medio de una estructura de metadatos. A parte, dicha estructura permite mantener una relación con otros procesos como archivo, uso, reutilización, etc.

$\mathrm{Su}$ estructura se basa en tres entidades primarias (personas, creaciones y acuerdos), más cuatro entidades secundarias o de apoyo (eventos, tiempo, lugar y derechos), que junto a ocho nexos entre ambos conforman una estructura en la que se da cabida a todos los metadatos imaginables para la gestión de los derechos.

\section{SMEF (Standard Media Exchange Framework)}

Creada por la BBC (British Broadcasting Corporation) como una herramienta para integrar sus propios sistemas de información y contenido audiovisual. La estructura de metadatos de SMEF cubre la información relacionada con los programas desde que son un proyecto, hasta que son difundidos en antena. Dicha estructura permite que los metadatos se asignen una única vez, ya que la información, una vez creada y explotada, puede reutilizarse facilitando procesos posteriores como la identificación de material de archivo para otros programas.

De Jong apunta que este formato no puede utilizarse de manera gratuita, pero sí el llamado Open SMEF que define el conjunto mínimo de metadatos para el intercambio de información entre organizaciones. Por último, decir que la BBC propone utilizar como base un lenguaje de etiquetado tal como XML del que ya hemos hablado anteriormente.

\section{ONTOLOGÍA ABC}

Aunque centrado en el mundo de las bibliotecas y archivos, fue diseñado para el tratamiento de los objetos digitales o analógicos con el proyecto Australian Harmony 
Digital Library. Esta propuesta plantea una estructura de base para que futuras comunidades planteen la suya propia. Dicha estructura se basa en las propuestas anteriormente descritas $<$ INDECS $>$ e FRBR, además de apoyarse en la semántica de RDF (Resource Description Frameworks).

El modelo $\mathrm{ABC}$ describe entidades y relaciones comunes además de entidades abstractas, temporales. Hay tres tipos de entidades, como son (De Jong, 2003):

- Temporalidad: expresa el estado en el que las propiedades del objeto existen.

- Realismo: abarca categorías que son tangibles y concretas.

- Abstracción: expresa conceptos e ideas, vinculados a la realización y a la manifestación en la categoría realismo.

A su vez, cada entidad se subdivide en clases y subclases cada una con sus respectivas propiedades conformando un núcleo con el que poder describir el contenido de los objetos de cualquier colección.

A parte de estas propuestas podríamos mencionar otras de manera más reducida, como son:

\section{VIDEO ACTIVE}

Proyecto que tiene como fin el dar acceso a los fondos audiovisuales de varias cadenas de televisión europeas. Con ello se pretende demostrar la evolución y características, tanto de la televisión como de la cultura, de los países que conforman la Unión Europea. Esta idea nació dentro del marco del proyecto eCONTENTPlus de la Unión Europea (cuya idea principal fue la de hacer accesible el contenido digital de la Unión Europea, además de favorecer su uso y explotación) y pretende dar acceso a la información de estos centros por medio de una estructura creada con metadatos.

\section{ECHO (European CHronicles On-line).}

Proyecto financiado por la Unión Europea en el que se pretende crear una infraestructura software que pueda ser mantenida por los archivos audiovisuales con fondos digitalizados para dar acceso a las colecciones de recursos audiovisuales históricos de interés. Entre las características del sistema están la indización automática de las obras, lo que permite un acceso inteligente a las mismas y la extracción automática de los términos de indización durante el proceso. La estructura de metadatos usada se basa en la propuesta IFLA-FRBR; más concretamente, aúna el formato Dublin Core y MPEG-7 basado en RDF.

Está desarrollado por cuatro archivos nacionales audiovisuales, como son:

- The Netherlands Institute for Sound and Vision.

- Memoriav (Suiza).

- Institut National de l'Audiovisuel (INA) (Francia).

- Istituto di Luce (Italia).

Además cuenta con el respaldo de otras entidades audiovisuales y universidades europeas. 


\section{OPEN VIDEO DIGITAL LIBRARY}

Es una iniciativa norteamericana que proporciona acceso a vídeos de una gran variedad de fuentes, especialmente de aquellas oficinas gubernamentales norteamericanas (como la NASA).

El proyecto comenzó en 1998 con el desarrollo de una plataforma para digitalizar una pequeña cantidad de material audiovisual. Con el paso del tiempo el fondo fue aumentando, al igual que las expectativas del proyecto.

Actualmente vemos que desde su página web se informa que se contiene material de las siguientes colecciones:

- Universidad de Maryland (Open House Video Reports).

- El Proyecto Informedia de la Universidad Carnegie Mellon.

- El Internet Moving Images Archive.

- 2001 TREC Video Retrieval Test Collection.

- CHI Video Retrospective.

- El Proyecto Digital Himalaya.

- Programa Educativo NASA K-16.

- La colección de William R. Ferris.

El Open Video Digital Library utiliza los metadatos para acceder y describir los recursos almacenados en los servidores y poder suministrarlos a los usuarios que lo soliciten. Tras el concepto de este proyecto vemos la idea de Open Archive Initiative, el cual suministra documentos en acceso libre a los usuarios. Estas nuevas formas de publicación se convierten en nuevos modos para que la documentación sea transmitida fuera de los tradicionales caminos a los que hasta ahora estábamos acostumbrados.

Por tanto, como hemos podido ver con esta introducción a los metadatos dentro del ámbito de lo audiovisual, podemos decir que estos garantizan que el usuario pueda tener acceso a los datos, junto con toda la información necesaria para entender e interpretarlos. Además de que gracias a las redes de trabajo estos metadatos pueden estar accesibles desde cualquier punto de la organización.

\section{ESQUEMAS DE METADATOS EN AUDIOVISUAL}

Todas las entidades que trabajan con material audiovisual observan el esfuerzo que conlleva el crear una estructura de metadatos de manera efectiva. Por ello se prefiere el compartir el trabajo y basar sus diseños en normas ya existentes, aunque los estándares no cubran todas las necesidades de estas organizaciones (De Jong, 2003).

Esto se debe a que normalmente los modelos cubren un aspecto en concreto del dominio audiovisual y no facilitan su adaptación a otro. Además, la variedad de objetivos, requisitos, procedimientos y necesidades de los usuarios de una empresa audiovisual hace complicado la creación de un modelo común de metadatos para toda la entidad. 
Los esquemas de metadatos son principalmente desarrollados por organismos internacionales (como la ISO o EBU -European Broadcast Union) y organismos audiovisuales dentro de proyectos o iniciativas continentales orientados al contenido digital en internet. Destaca la participación de canales de televisión o archivos audiovisuales nacionales dentro de proyectos continentales o iniciativas propias, como es el caso del archivo audiovisual nacional holandés (Netherlands Institute for Sound and Vision) que trabaja desde hace unos años en diferentes proyectos, tanto nacionales como internacionales, encaminados a mejorar el acceso y la conservación del patrimonio audiovisual.

El resultado de estos proyectos es la creación de un estándar de metadatos. La información relativa a los mismos suele aparecer en páginas web mantenidas por la organización responsable de su desarrollo en la que encontramos principalmente información teórica, echando en falta testimonios de posibles aplicaciones prácticas llevadas a cabo dentro del proyecto que nos den una visión real del alcance del mismo.

Por otro lado estos estándares necesitan de utilidades que definan el interfaz sobre los que se transferirán; hablamos de los estándares de intercambio de datos. Éstos garantizan que la información sea transferida de manera que no varíe la información contenida en los metadatos. Hablaremos brevemente de tres de ellos: XML, RDF y KLV.

XML (eXtensible Mark-up Language), del que hemos hablado en el capítulo anterior, es uno de los metalenguajes más conocidos que nos permite traducir cualquier clase de metadatos a un formato de representación común para que sea transferido entre sistemas y a través del web. Uno de los puntos fuerte de XML es que distingue entre forma y contenido, además de que nos permite que los metadatos puedan leerse e interpretarse aún cuando los sistemas usados en la transferencia sean diferentes.

Otro de los estándares de intercambio de los que más se está hablando en los últimos años por sus características es RDF (Resource Description Framework). Permite intercambiar metadatos de manera global, sin importar el sistema utilizado y nos sirve de base para desarrollar nuevas normas. Hay autores que lo consideran como metamodelo de metadatos (Eva Méndez, 2002) ya que define un modelo de metadatos de alto nivel con una sintaxis expresada en XML. Como decimos, está ganando cada día más fuerza debido a que permite que cada comunidad utilice su propio vocabulario de metadatos, lo cual no repercute a la hora del intercambio de la información, ya que al basarse en XML, permite esta acción; por otra parte los documentos RDF son fáciles de manejar ya que se basan en una estructura de tres partes:

- Un recurso: todo aquello que pueda tener una URL.

- Una propiedad: una característica de un recurso.

- Una declaración: consiste en la unión de un recurso, una propiedad y un valor.

Por último hablaremos de KLV (Key Length Value) que es el ejemplo más claro de protocolo de intercambio dentro del mundo audiovisual. Este facilita de modo específico la codificación de metadatos y materiales digitales entre sistemas de producción. KLV es un elemento de un sistema media que soporta la codificación de 
los metadatos, de audio, vídeo y materiales multimedia para varias normas de comprensión como las MPEG.

El protocolo se basa en las definiciones dadas por SMPTE (Society of Moving Pictures and Television Engineers) e ISO pero puede adaptarse a otros esquemas de metadatos.

Para terminar este apartado, comentar que además de estos tres lenguajes descritos, la mayoría de estándares se basan en Dublin Core, debido a su facilidad de uso y a las prestaciones que traen consigo en la descripción de material audiovisual. En el siguiente punto trataremos el formato centrado en los documentos audiovisuales y veremos cómo otros estándares se basan en su estructura para mapear o utilizarlo como referencia para crear sus esquemas de metadatos.

\section{PRINCIPALES ESTÁNDARES DE METADATOS SOBRE LO AUDIOVISUAL}

Una vez visto cómo podemos intercambiar los datos, nos centraremos en los estándares de metadatos más importantes dentro del mundo audiovisual. Podemos decir que al igual que en otros campos, en lo relativo a lo audiovisual se están desarrollando en las últimas décadas importantes estándares sobre metadatos, aunque hay autores que opinan que estos no están a la altura y que no permiten describir los documentos audiovisuales con calidad (De Jong, 2003).

Los estándares de metadatos audiovisuales apoyan la producción, distribución y archivos de materiales de vídeo y audio. Y como veremos, se dirigen hacia los procesos de captura, creación, producción, descripción e identificación de contenidos, protección de derechos, intercambio, distribución y consumo (Orozco García-Mayorca, 2001). Intentando definir de los metadatos:

Su estructura y sintaxis.

Sus relaciones.

Cómo los podemos codificar para su transmisión.

Definir su difusión.

Normalizar cómo acceden a ellos las aplicaciones.

Dicho esto, pasamos a comentar algunas de las experiencias internacionales sobre los metadatos audiovisuales. Trataremos las siguientes iniciativas:

\subsection{AAF (Advanced Authoring Format)}

Desde febrero de 2007 ha cambiado se denominación y se le conoce como Advanced Media Workflow Association (AMWA). Este formato nació para mejorar el intercambio de información sobre imágenes, sonido y metadatos por medio de plataformas y aplicaciones. Puede trabajar con grandes colecciones de metadatos y documentos audiovisuales siempre en la etapa de creación, ya que no cubre otras como la distribución o almacenamiento. 
Este formato es "capaz de permitir todas las necesidades de almacenamiento durante el proceso dinámico de la creación” (De Jong, 2003). Entre sus características está que evita que se tengan que transferir imágenes, texto, audio o vídeo procedentes de fuentes diferentes a formatos que puedan ser utilizadas por distintas aplicaciones.

Referente a su forma de trabajar los metadatos de AAF permiten modificar a otros metadatos con lo que se pueden definir nuevas clases con las que ampliar la descripción de un documento. Por otra parte, el software AAF nos permite gestionar conjuntos de metadatos, definir perfiles de usuario o conectar módulos.

Los puntos principales de este estándar son:

La especificación de objeto AAF.

La especificación de estructura de bajo nivel (que describe cómo se almacenan los objetos).

Desarrollo del software AAF que posibilita las aplicaciones cliente para acceder a los datos almacenados en un fichero AAF.

Este estándar se basa en el esquema creado con SMPTE. Y además mantiene relaciones con otro de los esquemas que más adelante trataremos, MXF. AAF presenta dificultades a la hora de la aplicación cuando tratamos información proveniente de cámaras o grabadoras, por tanto, la Asociación AAF desarrolla el formato MXF para permitir intercambiar la información proveniente de diferentes medios.

\subsection{DUBLIN CORE}

El 6 de octubre de 2000, en el marco del $8^{\circ}$ Workshop de la Dublin Core en Ottawa (Canadá) se creó el Grupo de Interés especializado en archivos de imágenes.

Hasta entonces la Dublin Core (DC) se había centrado en suministrar metadatos orientados en documentos textuales, pero con este grupo se intentó normalizar los metadatos sobre documentos audiovisuales. El primer propósito de este grupo fue el recoger las propuestas e iniciativas que se estaban llevando a cabo para ver cómo la Dublin Core se adaptaba y utilizaba para documentos audiovisuales. Tras esto llevaron a cabo varias iniciativas entre las que destaca la realizada en colaboración con el Australian Center for Moving Image (anteriormente denominado Cinemedia) para catalogar sus fondos audiovisuales y ponerlos accesibles con la ayuda del esquema de la Dublin Core expresados en XML.El esquema Dublin Core ha servido de base a los demás estándares que se encargan de tratar los documentos audiovisuales con metadatos; ejemplos los tenemos en los formatos MPEG 7 y 21, SMPTE, P-META y P-FRA. El caso de este último llama la atención por estar basado en su totalidad en Dublin Core. El resto, o han mapeado el formato o se han basado en su estructura.

Uno de los ejemplos más claros de adaptación de la DC a documentos audiovisuales es el proyecto ViDE (Video Devepolment Initiative), el cual "trata de realizar un perfil de aplicación para el vídeo digital, el cual comprende metadatos de tipo administrativo y estructural" (López, Sánchez, Pérez, 2003). Por tanto, vemos que 
utiliza el formato Dublin Core como base para describir la creación, difusión, el acceso y el almacenamiento de documentos audiovisuales en formato digital.

El grupo de trabajo dedicado a las imágenes en movimiento desapareció tras la Conferencia Internacional de la Dublin Core celebrada en Tokio en el año 2001.

\subsection{LISTA DE DATOS MÍNIMOS DE LA FIAT-IFTA}

No se puede considerar como un estándar de metadatos como los hasta ahora descritos, ya que no es un formato legible por máquinas, pero aporta todos los elementos necesarios para describir el contenido de los documentos audiovisuales (vídeos y otros materiales fílmicos en particular) en todos los aspectos: contenido, derechos, características físicas, etc.

Esta lista fue creada en 1986 antes de la llegada de Internet a los archivos audiovisuales y proporciona una estructura de campos o metadatos que definir los documentos audiovisuales.

Los campos los podemos agrupar en:

Identificación (datos para individualizar al documento en la colección). Datos técnicos (descripción del contenido y forma del documento).

Derechos de uso.

La importancia de esta lista en los posteriores estándares de metadatos audiovisuales la encontramos cuando esquemas de la importancia de SMPTE la utilizan para mapearla y crear su propia estructura de metadatos.

No obstante, cuenta con una serie de peculiaridades que lo hacen importante pero no completo. Esta estructura lógica de base de datos está adaptada para la conservación y almacenamiento de programas no informativos, quedando fuera las necesidades y requerimientos para este otro tipo de producto audiovisual televisivo. Además, los requerimientos actuales son mucho más complejos que los que se demuestra en esta estructura, resultando ambigua y especialmente obsoleta y mínima.

\subsection{METS (Metadata Encoding and Transmission Standard)}

Formato elaborado por la Digital Library Federation y mantenido por la Biblioteca del Congreso que nació a principios del siglo XXI como continuación de los trabajos llevados a cabo dentro del proyecto MOA2 (Making Of America II) que era un banco de pruebas de iniciativas orientadas a las bibliotecas digitales.

"Se trata de un lenguaje basado en XML destinado a proporcionar la sintaxis necesaria para le gestión de metadatos en bibliotecas digitales, facilitando la gestión e intercambio de documentos" (López, Sánchez, Pérez, 2003).

METS está principalmente pensado para el intercambio de archivos audiovisuales que contengan imágenes, vídeos o sonidos ubicados en una biblioteca digital.

$\mathrm{Su}$ estructura se basa en 7 secciones, como son: 
- Información sobre el documento METS, como la fecha, nombre del creador, etc.

- Metadatos descriptivos que analizan el objeto al que se hace referencia en un documento METS. Esta descripción suele basarse en Dublin Core.

- Metadatos blinadministrativos que contienen información sobre el documento original orientada a conocer el formato, derechos, origen digital de los datos y los ficheros que conforman el documento.

- Los ficheros que comprenden la versión electrónica del objeto digital.

- La estructura jerárquica del objeto para navegar por él.

- Hipervínculos entre la estructura jerárquica.

- Y una última sección que se usa para asociar comportamientos ejecutables con los contenidos del objeto METS.

\subsection{MPEG (Moving Picture Experts Group)}

Es uno de los formatos que más aceptación está teniendo en los últimos años. Es un estándar ISO para la descripción de recursos audiovisuales válido para utilización por parte de humanos como por máquinas.

Se basa en los antiguos estándares de codificación para el vídeo interactivo en cdrom, el DVD y la televisión digital (MPEG-1 y 2) y el estándar de codificación para documentos multimedia basado en objetos (MPEG-4).

Tenemos que señalar que no se trata de un esquema de metadatos como los demás descritos, sino un medio para representar los documentos audiovisuales de diversa índole, tales como imágenes estáticas, imágenes en movimiento, vídeo, audio o documentos que combinan algunos de ellos.

Para llevar a cabo su misión establece un conjunto de descriptores para representar los documentos y una serie de esquemas que proporcionan la estructura semántica y las relaciones entre los descriptores. Esto es posible gracias a una DDL (siglas en inglés de Lenguaje de Definición de Datos) que básicamente es una adaptación de XML a los documentos multimedia.

Nos centraremos en dos de los formatos más actuales: MPEG-7 y MPEG-21.

La investigación centrada en MPEG-7 está orientada en los aspectos de producción y archivo del material para servirlo posteriormente. Una de las características que presenta este formato es que muestra una serie de descriptores relacionados únicamente con un tipo de documento, así tenemos descriptores y esquemas relacionados con documentos visuales, de audio y para la descripción de otros documentos multimedia.

Este formato lo podemos ver relacionado con otros, tal como SMPTE, TV-Anytime o P-Meta. A principio de siglo se creó un grupo de trabajo dedicado a integrar MPEG en otros formatos, así se mapeó SMPTE y MPEG-7. Además se trabaja en aunar Dublin Core y este formato mediante XML lo que permitirá búsquedas sencillas con Dublin Core y más específicas con MPEG.

El otro formato del que hablaremos es MPEG-21, que tiene por objetivo el definir un marco abierto para aplicaciones multimedia. Específicamente, MPEG-21 define un estándar común en el entorno multimedia para la distribución o el consumo y definir la 
tecnología necesaria para que el usuario pueda intercambiar, acceder o manipular la información mediante la descripción de los elementos que entran en juego en la red. Se trata de un sublenguaje de XML que está diseñado para comunicar información de licencias en un formato utilizable automáticamente.

En palabras de De Jong (2003) este formato:

"Integrará las tecnologías críticas dentro del campo audiovisual y multimedia, tratando de hacer realidad la inexistente "imagen completa" (donde estén en un mismo entorno, todos los sistemas y elementos relacionados con los procesos de producción, distribución y archivo)".

MPEG 21 aún está en proceso de pruebas y trabajando con el punto de vista puesto en una futura interacción con otras iniciativas, como son SMPTE, P-Meta, TVAnytime y Dublin Core.

\subsection{MXF (Material eXchange Format)}

Es otra iniciativa surgida del foro Pro-MPEG para crear un formato de fichero para la transferencia de tipos diferentes de materiales de programas multimedia entre el almacenamiento y el equipo del procesado. Es decir, MXF proporciona una estructura para empaquetar la información audiovisual (vídeo, audio y metadatos asociados) y poder enviarla o almacenarla.

Este formato presenta las siguientes características que le hacen válido como estándar:

Es independiente del sistema operativo, del sistema de comprensión y de las infraestructuras de red.

Los documentos MXF están pensados para su estructura secuencial y para su lectura secuencial y aleatoria.

La información puede transferirse como stream y convertirla luego en un formato estándar.

Usa el protocolo KLV.

De forma muy reducida, la tecnología stream es aquella que tiene como fin el difundir aquellos contenidos multimedia aprovechando las posibilidades de internet. El usuario final recibe una pequeña parte de la información almacenada en el servidor, la cual servirá de enlace o apoyo para la llegada de más información, con lo que se mejora la recepción de la información, ya que el usuario no necesita esperar la descarga completa del archivo, sino que puede visualizar el contenido tal y como se vaya recibiendo en su ordenador. En palabras de De Jong (2003) este estándar "facilita un equipamiento de televisión flexible, que pueda ser interoperable sobre distintas aplicaciones especificas de usuario, incluyendo aquellas especificadas por las normas SMPTE". 
Un documento MXF contiene una cabecera en la que se da información sobre el fichero de metadatos, un cuerpo que incluye al audio y vídeo, un pie de página con el que se acaba el documento.

Este estándar se basa en etiquetas SMPTE utilizando un modelo de metadatos basado en AAF, lo que permite realizar un mapeo entre ambos formatos. Con esto se consigue que documentos que aún no han sido tratados (como los brutos) sean almacenados en MXF, mientras que documentos relacionados con los efectos de composición son manejados con AAF.

Finalmente, podemos decir que se está trabajando en mejorar el estándar y hacerlo compatible con otros, así EBU está trabajando para adaptar MXF al formato P-Meta, mientras que el proyecto Pro-MPEG intenta enlazar al estándar con los MPEG.

\subsection{P-FRA (Proyecto Future Radio Archives)}

Enmarcado dentro del grupo de trabajo de EBU intenta definir un conjunto sencillo de metadatos adaptado para archivos sonoros. Para ello se basa en Dublin Core con la misma estructura que este, la cual proporciona tres tipos de información:

Una interpretación de los elementos con vistas a su difusión.

Cuando sea necesario, los metadatos podrán ser refinados para permitir un mayor detalle.

Se ofrece texto controlado para algunos elementos, contando así con una terminología común.

\subsection{P-META}

Es otro de los proyectos del grupo de trabajo de EBU que crea una norma para los elementos media y su intercambio entre las distintas áreas del proceso y las organizaciones mediante la construcción de un modelo de intercambio para la información sobre el material de programas.

Dentro de la norma se describen metadatos para la identificación, descripción, consulta y uso de los contenidos.

Este estándar se basa en el desarrollo de otro estándar creado por la BBC, como es SMEF (Standard Media Exchange) que está enfocado a la producción. La idea de PMeta es unir SMEF con la estructura de SMPTE para crear un marco único y universal para la descripción.

P-Meta es independiente del sistema y se puede utilizar en cualquier plataforma que utilice XML o KLV.

Otra característica de P-Meta es que se trabaja para mapearlo con MXF, lo que aumentará sus posibilidades de intercambio. Otras iniciativas de mapeo del formato que se plantearon fueron con Dublin Core y con TV-Anytime. 


\subsection{SMIL (Synchronized Multimedia Integration Language)}

Es una especificación de W3C (Consorcio World Wide Web, cuyo fin es desarrollar estándares web) a cargo del grupo de trabajo Synchronized Multimedia Activity $(S Y M M)$ basada en XML que permite describir documentos multimedia aumentando dicha descripción con la integración o asociación de información en diversos formatos.

Por otra parte también permite adaptarse a otras propuestas surgidas de XML como $\mathrm{RDF}$, lo que aumenta su potencia de descripción.

Entre las ventajas de SMIL está la integración de metadatos y de una sintaxis RDF o XML lo que "permite un marco de descripción de documentos unificado y a la vez extensible, permitiendo la perfecta integración de vocabularios controlados dentro del documento" (Pérez, Sánchez y Caldera, 2004).

Otra de las ventajas de SMIL es que muchos de los reproductores más conocidos, como Real Player, Windows Media o Quick Time, lo aceptan y soportan.

\subsection{SMPTE (Society of Moving Pictures and Television Engineers)}

El grupo de trabajo de SMPTE ha tenido como misión la elaboración de un estándar de metadatos centrados en los procesos de producción de material audiovisual en entornos televisivos. Así surgió el estándar del que hablamos, que puede utilizarse con independencia del sistema de producción utilizado por una empresa.

Este esquema intenta cubrir todo el proceso de producción, abarcando la pre y postproducción, compra, distribución, transmisión, almacenamiento y archivo.

Tiene una estructura jerárquica basada en KLV compuesta por diez categorías más una añadida en la que se encuentran aquellos metadatos que aún no se sabe dónde ubicarlos. Además utiliza XML para la representación de la información.

Este estándar ha servido de base para diferentes propuestas descritas anteriormente y además ha sido mapeado por otros tantos estándares. Propuestas como la AAF o MXF se basan prácticamente en el esquema de SMPTE. Se han llevado a cabo mapeos entre distintos elementos de otros estándares ya definidos anteriormente en P-Meta y MPEG-7.

\subsection{TV-ANYTIME}

Es una iniciativa surgida en 1999 por la unión de varias empresas de multidifusión. Tiene como fin crear una estructura de metadatos que facilite la descripción de programas de televisión con el fin de desarrollar guías de programación destinadas a usuarios y profesionales del medio interesados en la compra o intercambio de contenidos.

La idea principal de TV-Anytime es la búsqueda, selección, localización y adquisición de contenido en cualquier lugar o momento. Por otra parte, se ha diseñado un modelo de flujo de datos y un formato común de representación de metadatos para los distribuidores de contenidos digitales, que permite el desarrollo de aplicaciones o 
servicios complementarios como la televisión interactiva, los sistemas de guías a los padres, multilingüísmo, etc.

Este estándar ha estado vinculado a proyectos de documentación digital como MPEG o P-Meta, lo que "garantiza una completa adaptabilidad con el resto de los que se están desarrollando y lo convierte en un complemento de gran utilidad para este sector tan especializado" (López, Sánchez, Pérez, 2003). Además, las especificaciones de metadatos están muy influidas por las dadas por MPEG-7, aunque también está al tanto de lo que se realiza desde el proyecto SMPTE.

Por último decir que TV-Anytime trabaja con el lenguaje de definición de descripción (DDL) creado por MPEG-7 y que utiliza como medio de codificación, XML. Asimismo, se está trabajando en conjunto con P-Meta para el desarrollo de un esquema de clasificación de géneros para radio y televisión de TV-Anytime que intenta sustituir una versión ya anticuada.

\subsection{UMID (Unique Material IDentifier)}

La idea de este estándar es la de identificar de manera única un documento sin tener en cuenta el ámbito en el que nos encontremos. En el caso de la documentación televisiva, puede referirse tanto a conjuntos de contenidos ya elaborados como a brutos. Además, nos permite no sólo la identificación de los documentos a través de un código específico, sino también la relación entre los documentos y sus metadatos a través del código.

La estructura de UMID puede tener dos aspectos:

La denominada Basic UMID que proporciona información descriptiva del documento. Y Extended UMID, con la que conocemos datos relativos a su localización, información sobre los usuarios y fechas.

\section{CONCLUSIONES}

Después de ver las propuestas llevadas a cabo relacionadas con el tratamiento de la información audiovisual, vemos que son pocas las centradas en la televisión, pero aún lo son menos las que tratan los documentos analizados en los servicios informativos de televisión. Así podemos hablar de TV-Anytime y la Lista de datos mínimos de la FIAT. Siendo esta última un referente para los demás estándares surgidos con anterioridad sin llegar a ser una estructura de metadatos en si, como son el resto de propuestas descritas, pero conforma una estructura de campos que describen el documento de manera completa y efectiva para una posterior recuperación de la misma.

A modo de conclusión podemos decir que la era digital trae consigo toda clase de productos digitales, entre los que podemos destacar los recursos audiovisuales que proliferan por toda la red y archivos audiovisuales, tales como vídeos, imágenes, sonidos, etc. Las empresas televisivas o audiovisuales, además de otras organizaciones 
productoras, crean una gran cantidad de estos recursos, que para que sean recuperados por los usuarios o por profesionales necesitan ser organizados y representados mediante estructuras de descripción, como los metadatos, que adquieren gran importancia por su capacidad de descripción (ya que abarcan todo el proceso de producción audiovisual) y porque nos permite establecer niveles de usuarios.

\section{BIBLIOGRAFÍA}

AAF (Advanced Authoring Format). Disponible en: http://www.aafassociation.org/ [consultado en: 26-10-2009].

DE JONG, Annemieke (2003). Los metadatos en el entorno de la producción audiovisual. Méjico: [s.n.].

DUBLIN CORE. Disponible en: http://dublincore.org. [consultado en: 26-10-2009].

ECHO (European CHronicles On-line). Disponible en: http://pcerato2.iei.pi.cnr.it/echo/ [consultado en: 26-10-2009].

INDECS. Disponible en: http://www.indecs.org [consultado en: 26-10-2009].

KASHYAP, Vipul, SHETH, Amit (1997). Semantic heterogeneity in global information systems: the role of metadata, context and ontologies [en línea]. En: PAPAZOGLOU, Michael P.,

SCHLAGETER, Gunter. Cooperative information systems: current trends and directions. San Diego: Academic Press. Disponible en: http://citeseer.ist.psu.edu/295715.html. [consultado en: 03-10-2009].

LISTA DE DATOS MÍNIMOS DE LA FIAT-IFTA. Citado por: CALDERA SERRANO, Jorge (1999). "Análisis de las recomendaciones de la FIAT/IFTA sobre los datos mínimos a señalar en las bases de datos de los archivos de televisión" [en línea]. En: Cuadernos de documentación multimedia, $\mathrm{n}$ 8. Disponible en: http://www.ucm.es/info/multidoc/multidoc/revista/num8/caldera.html. [Consultado en: 12-10-2009].

LÓPEZ YEPES, Alfonso, SÁNCHEZ JIMÉNEZ, Rodrigo, PÉREZ AGÜERA, José Ramón (2003). "Tratamiento de la documentación audiovisual en el entorno digital: iniciativas de metadatos y lenguajes de descripción multimedia". En: El profesional de la información, vol.11. n 6, págs. 443-451.

MENDEZ RODRÍGUEZ, Eva Ma (2002). Metadatos y recuperación de información: estándares, problemas y aplicabilidad en bibliotecas digitales. Gijón: Trea, 2002 (Biblioteconomía y Administración Cultural).

METS (Metadata Encoding and Transmission Standard). Disponible en: http://www.loc.gov/standards/mets/ [consultado en: 26-10-2009].

MINGLIAN, Duan, XINGXING, Yao, JIUZHEN, Zhang (2004). "A study on audiovisual metadata" [en línea]. En: International Conference on Dublin Core and Metadata Applications, Shangai. Disponible en: http://www.slais.ubc.ca/PEOPLE/faculty/tennisp/dcpapers2004/Paper_17.pdf [consultado en: 26-10-2009]. 
MODELO IFLA-FRBR (Requerimientos Funcionales para Registros Bibliográficos). Disponible en: http://www.ifla.org/VII/s13/frbr/frbr.pdf [consultado en: 26-102009].

MPEG (Moving Picture Experts Group). Disponible en: http://www.chiariglione.org/mpeg/ [consultado en: 26-10-2009].

MXF (Material eXchange Format). Disponible en: http://mxf.info/ [consultado en: 2510-2009].

ONTOLOGÍA ABC. Disponible en: http://metadata.net/harmony/lagoze_hunter_dc2001.pdf [consultado en: 26-102009].

OPEN VIDEO DIGITAL LIBRARY. Disponible en: http://www.open-videotoolkit.org/index.php [consultado en: 26-10-2009].

OROZCO GARCÍA-MAYORCA, Alejandra (2001?). La construcción de metadatos en el proceso de organización, análisis documental y recuperación de la información en los archivos de imágenes en movimiento [en línea]. Disponible en http://www.patrimoniofilmico.org.co/docs/metadatos.pdf. [consultado en: 26-102009].

PÉREZ AGÜERA, José Ramón, SÁNCHEZ JIMÉNEZ, Rodrigo, CALDERA SERRANO, Jorge (2004). "Adaptación de tecnologías stream y XML a centros de documentación en televisión". En: Revista española de Documentación Científica, vol 27, n 4, págs. 441-454.

P-FRA (Proyecto Future Radio Archives). Disponible en: http://www.ebu.ch/tech_t3293.html.

P-META.

Disponible en: http://www.ebu.ch/en/technical/metadata/specifications/notes_on_tech3295.php [consultado en: 26-10-2009].

POCKLEY, Simon, CAVANAGH, Emily (2001). "Collaborative cataloguing of moving images and new media art works" [en línea]. En: International Conference on Dublin Core and Metadata Applications, Tokio. Disponible en: http://www.nii.ac.jp/dc2001/proceedings/product/paper-20.pdf. [consultado en: 0110-2009]

RÍOS HILARIO, Ana Belén (2006). "Diseño de un modelo de descripción para los recursos audiovisuales basado en el modelo FRBR" [en línea]. En: Proceedings VIII Jornadas de Gestión de la Información, Madrid. Disponible en: http://eprints.rclis.org/archive/00007898/. [consultado en: 01-10-2009].

SMEF (Standard Media Exchange Framework). Disponible en: http://www.bbc.co.uk/guidelines/smef/ [consultado en: 26-10-2009].

SMIL (Sinchronized Multimedia Integration Language). Disponible en: http://www.w3.org/AudioVideo/ [consultado en: 26-10-2009].

SMPTE (Society of Moving Pictures and Television Engineers). Disponible en: http://www.smpte.org/home [consultado en: 26-10-2009].

TV-ANYTIME. Disponible en: http://www.tv-anytime.org/ [consultado en: 26-102009]. 
UMID (Unique Material IDentifier). Citado por: LÓPEZ YEPES, Alfonso, SÁNCHEZ JIMÉNEZ, Rodrigo, PÉREZ AGÜERA, José Ramón (2003). En: “Tratamiento de la documentación audiovisual en el entorno digital: iniciativas de metadatos y lenguajes de descripción multimedia". El profesional de la información, vol.11. n. 6, págs. 443-451.

VIDEO ACTIVE. Disponible en: http://videoactive.wordpress.com/ [consultado en: 2610-2009]. 